\title{
LONGITUDINAL AND TRANSVERSE FEEDBACK KICKERS FOR THE BESSY II STORAGE RING*
}

\author{
S. Khan, T. Knuth ${ }^{\dagger}$, BESSY, Berlin, Germany \\ A. Gallo, F. Marcellini, B. Sparato, M. Zobov, INFN, Frascati, Italy
}

\section{Abstract}

This paper presents an overview of the bunch-by-bunch feedback kickers designed for the BESSY II storage ring. Simulation results for the longitudinal kicker cavity and for the transverse stripline kicker are discussed.

\section{INTRODUCTION}

Bunch-by-bunch feedback systems are required to cure multibunch beam instabilities at high currents in the BESSY II storage ring [1]. The present status of the longitudinal feedback system (LFB) and the transverse feedback system (TFB) is reported in [2]. As longitudinal correcting element, a waveguide-overloaded cavity was favored over a drift-tube structure. The kicker cavity designed for DA $\Phi N E[3]$ was modified to meet the requirements for BESSY II. A shunt impedance of $960 \Omega$ and effective damping of higher-order modes (HOMs) has been achieved. For the TFB, stripline electrodes will be used. The stripline pairs for each transverse plane will be combined in one structure.

This paper describes the design issues and focusses on simulation results for both kickers using the computer codes MAFIA [4], POISSON [5] and HFSS [6]. The parameters used in the simulations are given in Table 1.

Table 1: Simulation parameters for BESSY II.

\begin{tabular}{|c|l|l|}
\hline$f_{\mathrm{rf}}$ & rf frequency & $499.65 \mathrm{MHz}$ \\
$n$ & harmonic number & 400 \\
$E$ & beam energy & $1.7 \mathrm{GeV}$ \\
$I$ & assumed beam current & $400 \mathrm{~mA}$ \\
$P$ & total rf power (LFB) & $220 \mathrm{~W}$ \\
& total rf power (TFB) & $2 \times 150 \mathrm{~W}$ \\
\hline
\end{tabular}

\section{LONGITUDINAL KICKER}

\subsection{Geometry and Performance}

The LFB kicker is based on a pillbox cavity design as shown in Fig. 1. In order to achieve the desired bandwidth of [7]

$$
f_{\mathrm{bw}}=0.53 f_{\mathrm{rf}}=265 \mathrm{MHz},
$$

eight waveguides are attached to provide suitable damping. Four waveguides are used as power inputs, the four other waveguides are connected to loads.

\footnotetext{
* This work is funded by the Bundesministerium für Bildung, Wissenschaft, Forschung und Technologie and by the Land Berlin

† Email: knuth@bii.bessy.de
}

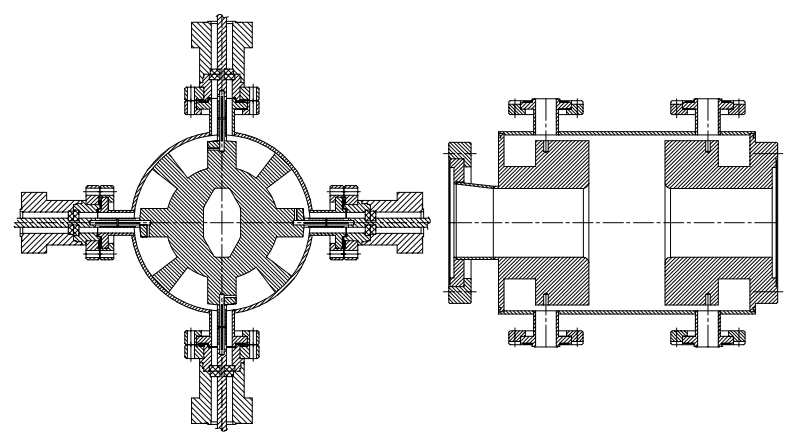

Figure 1: Longitudinal kicker structure.

MAFIA calculations yield an optimum $R / Q$-value of $87 \Omega$ at a cavity length of $1=72 \mathrm{~mm}$. The center frequency has to fulfill the condition [8]

$$
f_{c}=(p \pm 0.25) f_{r f}
$$

where $p$ is a positive integer. In order not to deviate too much from the original design, a center frequency of $1374 \mathrm{MHz}$ (i.e. $2.75 f_{\mathrm{rf}}$ ) was chosen, leading to a theoretical pillbox radius of $83 \mathrm{~mm}$. Extensive simulations were performed using the 3D-code HFSS in order to check the HOM content of the structure, to obtain the required $Q$ value of 5.2, and to optimize the transition from the coaxial feedthrough to the waveguide. Fig. 2 shows $1 / 8$ th of the kicker structure. The reflected power up to the cut-off frequency does not exceed 6\%. The port-to-port frequency response is shown in Fig. 3. With a center frequency of $1380 \mathrm{MHz}$ and a bandwidth of $270 \mathrm{MHz}$, a $Q$-value of 5.1 is obtained.

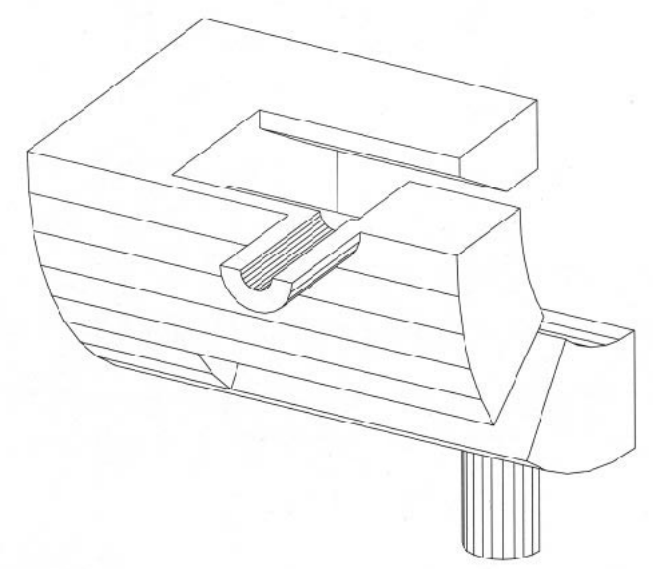

Figure 2: HFSS model as 1/8th of the whole structure. 


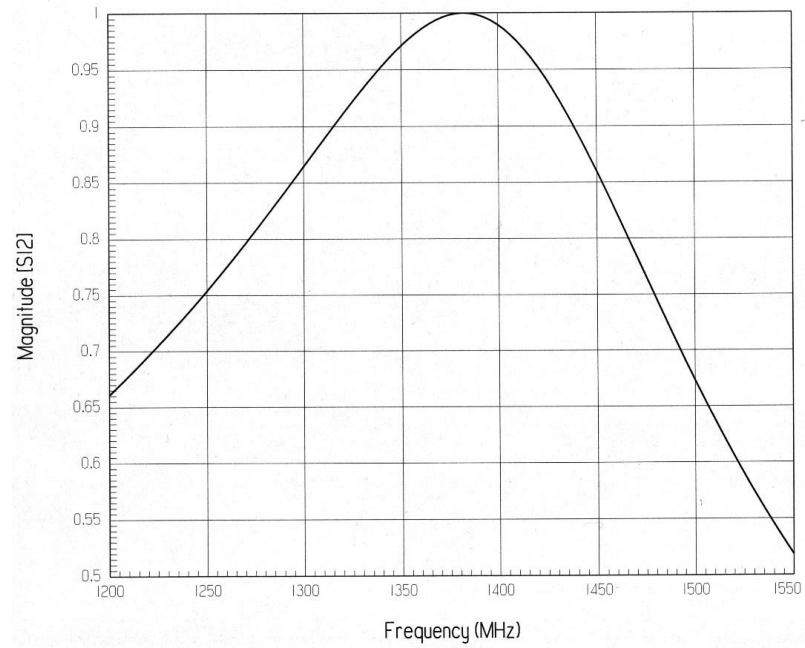

Figure 3: Frequency response of the fundamental mode.

For a given input power $P$, the simulation yields an electric field amplitude $E_{z}(z)$ and phase $\Theta_{z}(z)$ on the cavity axis, from which the accelerating voltage

$$
V_{\mathrm{acc}}=\int_{-l / 2}^{l / 2} E_{z}(z) \exp \left(i\left[2 \pi f_{c} z / c-\theta_{z}(z)\right]\right) d z
$$

is obtained. The resulting shunt impedance, given by

$$
R_{s}=\frac{\left|V_{\mathrm{acc}}\right|^{2}}{2 P}
$$

is $1100 \Omega$. An independent method to obtain $R_{s}$ by simulating a wire along the beam axis yields a slightly lower value of $960 \Omega$. Table 2 summarizes the kicker parameters.

Table 2: Longitudinal kicker parameters.

\begin{tabular}{|l|l|}
\hline pillbox length & $72 \mathrm{~mm}$ \\
length including waveguides & $260 \mathrm{~mm}$ \\
overall length & $310 \mathrm{~mm}$ \\
pillbox radius & $82 \mathrm{~mm}$ \\
number of waveguides & 8 \\
center frequency & $1380 \mathrm{MHz}$ \\
bandwidth & $270 \mathrm{MHz}$ \\
Shunt impedance & $960 \Omega$ \\
\hline
\end{tabular}

\subsection{HOM Characterization}

Monopole and dipole HOMs were selected using 2D MAFIA calculations, and their center frequencies were verified by $3 \mathrm{D}$ runs. In the vicinity of these frequencies, HFSS runs were performed to obtain the $Q$-values and shunt impedances listed in table 3 and table 4 . The power generated by the monopole modes was computed up to the cut-off frequency of $5.2 \mathrm{GHz}$, where each mode contributes a power of

$$
P_{G}=\sum_{i} \frac{1}{2} \operatorname{Re}\left[R_{c}\left(\omega_{i}\right)\right] I_{i}^{2}
$$

with $I_{i}$ representing the beam spectrum and $R_{c}$ the coupling impedance, which turned out to be twice the shunt impedance. Table 5 lists the total power contribution of the fundamental mode and the total power of all HOMs for beam currents of $200 \mathrm{~mA}$ and $400 \mathrm{~mA}$ in 320 buckets. Since the cavity is a non-directional device, about half of the generated power is seen by the amplifier i.e. $130 \mathrm{~W}$ at $400 \mathrm{~mA}$. A circulator for protection is not immediately needed, since the amplifier is capable of absorbing up to $100 \%$ of its output value $(220 \mathrm{~W})$.

Table 3: Monopole modes of the kicker cavity.

\begin{tabular}{|c|c|c|c|c|c|}
\hline Mode & $\begin{array}{c}f_{\text {MAFIA-3D }} \\
{[\mathrm{MHz}]}\end{array}$ & $\begin{array}{c}R_{s} / Q \\
{[\Omega]}\end{array}$ & $\begin{array}{c}f_{\mathrm{HFSS}} \\
{[\mathrm{MHz}]}\end{array}$ & $Q$ & $\begin{array}{c}R_{s} \\
{[\Omega]}\end{array}$ \\
\hline \hline 0 & 1405.8 & 86.9 & 1382 & 5.2 & 887 \\
\hline 1 & 2518.5 & 13.9 & 2250 & 7.1 & 99 \\
\hline 2 & 3231.1 & 2.6 & 3257 & 65 & 169 \\
\hline 3 & 3872.5 & 13.6 & 3849 & 149 & 2026 \\
\hline 4 & 4379.7 & 2.6 & 4195 & 32 & 83 \\
\hline
\end{tabular}

Table 4: Dipole modes of the kicker cavity.

\begin{tabular}{|c|c|c|c|c|c|}
\hline Mode & $\begin{array}{c}f_{\text {MAFIA-3D }} \\
{[\mathrm{MHz}]}\end{array}$ & $\begin{array}{c}R_{s}^{\perp} / Q \\
{[\Omega]}\end{array}$ & $\begin{array}{c}f_{\text {HFSS }} \\
{[\mathrm{MHz}]}\end{array}$ & $Q$ & $\begin{array}{c}R_{s}^{\perp} \\
{[\Omega]}\end{array}$ \\
\hline \hline 1 & 2190.5 & 4.32 & 2176 & 15 & 64.8 \\
& 2082.8 & & 2062 & 18 & 77.8 \\
\hline 2 & 2279.7 & 0.07 & 2257 & 30 & 2.1 \\
& 2108.0 & & 2085 & 97 & 6.8 \\
\hline 3 & 2968.4 & 3.3 & 3019 & 18 & 59.4 \\
\hline
\end{tabular}

Table 5: Total beam induced power with 320 buckets filled.

\begin{tabular}{|c|c|c|c|}
\hline$I$ & $P_{\text {fund. }}[\mathrm{W}]$ & $P_{\Sigma H O M}[\mathrm{~W}]$ & $P_{G}[\mathrm{~W}]$ \\
\hline \hline $200 \mathrm{~mA}$ & 35.5 & 29.9 & 65.4 \\
\hline $400 \mathrm{~mA}$ & 142.0 & 119.5 & 261.5 \\
\hline
\end{tabular}

\section{TRANSVERSE KICKER}

\subsection{Geometry and Performance}

For the transverse kicker, a stripline geometry will be employed. The horizontal $(x)$ and vertical $(y)$ electrodes are combined in a single structure to minimize space requirements and to obtain a moderate loss factor. Each pair of electrodes is driven in differential mode using a $180^{\circ}$ power divider connected to a $150 \mathrm{~W}$ linear amplifier.

Fig. 4 shows the model of the kicker for MAFIA calculations. C-shaped electrodes for the $x$-plane and flat electrodes for the $y$-plane match the octagonal shape of the adjacent vacuum chamber without tapering, leaving only a $5 \mathrm{~mm}$ wide gap in longitudinal direction. The electrode length of $300 \mathrm{~mm}$ maximizes the shunt impedance. To improve radiative heat dissipation, the outside surface is increased by adding cooling vanes. Using the $2 \mathrm{D}$ code POISSON, the electrodes and the surrounding chamber were shaped to meet the line impedance requirement of $R_{L}=50 \Omega$. A model was built and TDR (time domain 


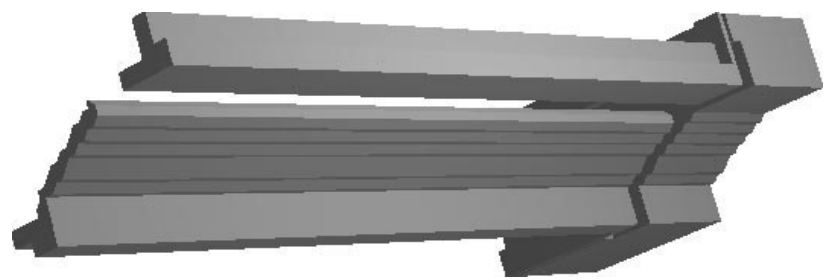

Figure 4: MAFIA model of the transverse kicker (1/8th of the full structure).

reflectometry) measurements were performed to verify the line impedance of the electrodes, which agrees well with the calculations, and to minimize reflections at the transition to the coaxial feedthroughs. The different geometry of the electrodes in $x$ and $y$ leads to a different transverse shunt impedance $R_{s}^{\perp}$ [9]

$$
R_{s}^{\perp}=2 R_{L}\left(g_{x, y} \frac{2}{k h}\right)^{2} \sin ^{2} \theta,
$$

where $g_{x, y}$ is the respective geometric coverage factor, $k$ is the wavenumber, $l$ is the electrode length, $h$ is the distance between opposite electrodes and $\theta=k l$. Fig.5 shows the frequency dependence of the shunt impedance in both planes.

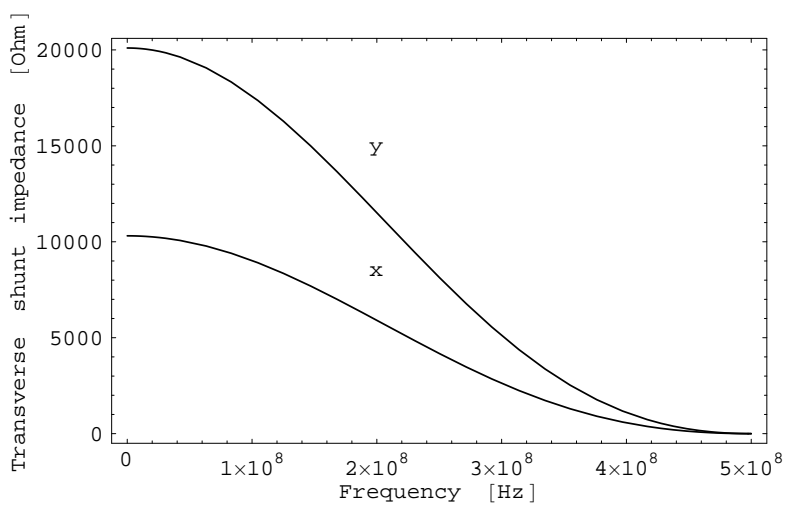

Figure 5: Shunt impedance of the transverse stripline kicker.

A higher vertical shunt impedance is prefered because of the larger vertical resistive wall impedance of a flat vacuum chamber. Over the entire mode spectrum (DC to $250 \mathrm{MHz}$ ), the kick voltage exceeds $1.7 \mathrm{kV}$ vertically and $1.2 \mathrm{kV}$ horizontally.

\subsection{HOMs and Power Losses}

HOMs found by performing MAFIA calculations in the frequency domain are trapped behind the electrodes and couple only weakly to the beam. Even though HFSS calculations show that most of the HOM power dissipates through the feedthroughs, at least one damping loop will be installed to further damp the the strongest modes.

Ohmic losses from the image currents passing the electrodes were calculated for a beam current of $400 \mathrm{~mA}$. A total power loss of $5 \mathrm{~W}$ for stainless steel (specific resistivity $\left.\varrho_{\mathrm{St}}=0.71 \cdot 10^{-6} \Omega \mathrm{m}\right)$ and $0.8 \mathrm{~W}$ for copper electrodes $\left(\varrho_{\mathrm{Cu}}=0.017 \cdot 10^{-6} \Omega \mathrm{m}\right)$ was obtained. On the other hand, using the Stefan-Boltzmann law and the emission coefficients for steel $\left(\epsilon_{\mathrm{St}}=0.29\right)$ and copper $\left(\epsilon_{\mathrm{Cu}}=0.03\right)$ electrode temperatures of $T_{\mathrm{St}}=80^{\circ} \mathrm{C}$ and $T_{\mathrm{Cu}}=105^{\circ} \mathrm{C}$ were found. In order to decrease the electrode temperature, the possibility of blackening the electrode surfaces is being considered

Table 6: Transverse kicker parameters.

\begin{tabular}{|l|l|}
\hline line impedance & $50 \Omega$ \\
length of kicker structure & $310 \mathrm{~mm}$ \\
overall length & $600 \mathrm{~mm}$ \\
(incl. bellow and pumping port) & \\
electrode separation $(x, y)$ & $65 \mathrm{~mm}, 35 \mathrm{~mm}$ \\
Coverage factor $(x, y)$ & $1.1,0.83$ \\
Kick voltage at DC $(x, y)$ & $1.7 \mathrm{kV}, 2.4 \mathrm{kV}$ \\
Kick voltage at 250 MHz $(x, y)$ & $1.2 \mathrm{kV}, 1.7 \mathrm{kV}$ \\
\hline
\end{tabular}

\section{ACKNOWLEDGMENTS}

The authors from BESSY would like to thank the members of the DA $\Phi N E$ rf group (INFN, Frascati) for their guidance in redesigning the kicker cavity. Valuable information for the layout of the transverse kicker was provided by $\mathrm{W}$. Barry and J. Corlett (LBNL, Berkeley).

\section{REFERENCES}

[1] R. Bakker for the BESSY team: 'Status and Commissioning Results of BESSY II', this conference.

[2] S. Khan, T. Knuth: 'BESSY II Feedback Systems', this conference.

[3] R.Boni et al.: 'A Waveguide Overloaded Cavity as Longitudinal Kicker for the DA $\Phi$ NE Bunch-by-Bunch Feedback System', Part. Acc. Vol.52 (1996), p.95.

[4] 'MAFIA User's Guide', CST, Darmstadt.

[5] J. H. Billen: 'The Superfish Manual', Los Alamos LA-UR96-1834.

[6] Hewlett-Packard Co.: 'HFSS, the High Frequency Structure Simulator HP85180A ${ }^{\mathrm{TM}}$,

[7] A. Gallo et al.: 'Efficiency of the Broadband Rf Cavity Longitudinal Kicker in DA $\Phi$ NE', internal note.

[8] M.Bassetti et al.: 'DA $\Phi$ NE Longitudinal Feedback', Proceedings of the 3rd Europ. Part. Acc. Conference (1992), p.807.

[9] D. Goldberg, G. Lambertson: 'Dynamic Devices: A Primer on Pickups and Kickers', AIP Conf. Proc. No. 249 (1992), p. 537. 